\title{
Recurrent neck abscesses due to cervical tuberculous lymphadenopathy in an elderly woman post-splenectomy: a case report
}

\author{
Aaron L Niblock
}

\begin{abstract}
Introduction: There are approximately 7000 new cases of tuberculosis every year in the UK, the majority of which are pulmonary. Approximately 5\% affect the lymph nodes in immunocompetent patients. Scrofula is an old term used to describe lymph nodes of the neck infected with tuberculosis
\end{abstract}

Case presentation: In the elderly population, growing neck lumps are always treated as red flags until a diagnosis is confirmed. Here, the case of an 89-year-old Caucasian woman is presented. She was reluctant to seek medical help as she feared the cause was sinister and did not want surgical intervention.

Conclusion: It is difficult to culture tuberculosis from superficial swabs, resulting in a high proportion of false negative results. Where there is a high degree of clinical suspicion for tuberculosis, it is important to consider a biopsy with culture. Patients over the age of 65 have waning immunity and are therefore a vulnerable group for acute infections as well as the re-activation of indolent organisms. Post-splenectomy patients are at a major disadvantage during sepsis and when a cellular immune response is required, such as when faced with a Mycobacterium tuberculosis infection. Scrofula is treated with a similar regime as pulmonary tuberculosis and has a near $100 \%$ success rate.

\section{Introduction}

In the elderly population, growing neck lumps are always treated as red flags until a diagnosis is confirmed. The case of an elderly patient presenting with neck lumps is here described. She was reluctant to seek medical help as she feared the cause was sinister and did not want surgical intervention. There are many laboratory tests used to diagnose tuberculosis (TB) infection however we rely on a high level of clinical suspicion.

Scrofula is an old term for TB affecting the lymph nodes of the neck, a more meaningful term is cervical tuberculous lymphadenopathy. It is usually the result of a primary infection of the lymph nodes with Mycobacterium tuberculosis. The bacteria can be spread by the lymphatic system or blood. Therefore, it could originate from a primary pulmonary focus. In adults, it is usually M. tuberculosis and in children, nontuberculous mycobacteria [1].

Correspondence: aaronniblock@doctors.net.uk

Royal Victoria Hospital, Medical Department, 274 Grosvenor Road, Belfast BT12 6BA
Scrofuloderma results from the breakdown of skin overlying a tuberculous focus, usually at a lymph node but also where skin overlies infected bones or joints. In the past, milk was frequently contaminated with $M$. bovis that resulted in a high number of children presenting with scrofuloderma. The oral or tonsillar primary lesion could progress to cervical adenitis which in turn resulted in the formation of cold abscesses. Clinically, these lesions are firm painless nodules that gradually enlarge and suppurate, forming ulcers and sinus tracts in overlying skin. Spontaneous healing can occur but often takes years and is often accompanied by the formation of hypertrophic scars. As in this case, the most common presentation of scrofula is a painless, often suppurative abscess that shows no signs of calor or erythema unless there is a secondary infection. Patients less frequently present with systemic signs such as weight loss and night sweats [2].

In Europe, with the rapid decrease of TB in the second half of the twentieth century, scrofula has become a very rare disease. The marked decrease in prevalence

\section{Biomed Central}


amongst the more economically developed countries was due to the pasteurization of milk and the Bacillus Calmette-Guérin vaccine. TB is still a major problem across the less economically developed countries, and therefore clinicians have a higher degree of suspicion when assessing patients.

Every year in the UK, it is estimated that there are 7000 new cases of TB. According to a study carried out in India, extrapulmonary TB constitutes about $15 \%$ to $20 \%$ of new TB cases in immunocompetent patients. These extrapulmonary sites are demonstrated in Figure 1 . This can rise to over $50 \%$ in immunocompromised patients, especially in patients positive for the human immunodeficiency virus (HIV) [3].

\section{Body response to infection with $T B$}

TB is spread by droplets that are inhaled into the respiratory tract, passing through the pharynx. It most commonly affects the lungs although it is possible to trigger a response in the tonsillar region. How the host's immune system responds to $M$. tuberculosis has a major role in determining the clinical manifestations. Once the organism has reached the alveoli it has four potential fates [4]: the immune system may destroy the bacilli and the patient gains immunity; the bacilli multiple and cause disease, that is, pulmonary TB; the bacilli become dormant and never cause disease; or the patient may develop reactivation TB - active disease due to an existing impairment in the immune system through, for example, HIV, malignancy or malnutrition.

During the primary infection with $\mathrm{TB}$ and during any subsequent secondary active disease, the bacteria are spread by blood or through the lymphatic system to any part of the body. Usually the bacteria are destroyed by the immune system; however, they may concentrate at a

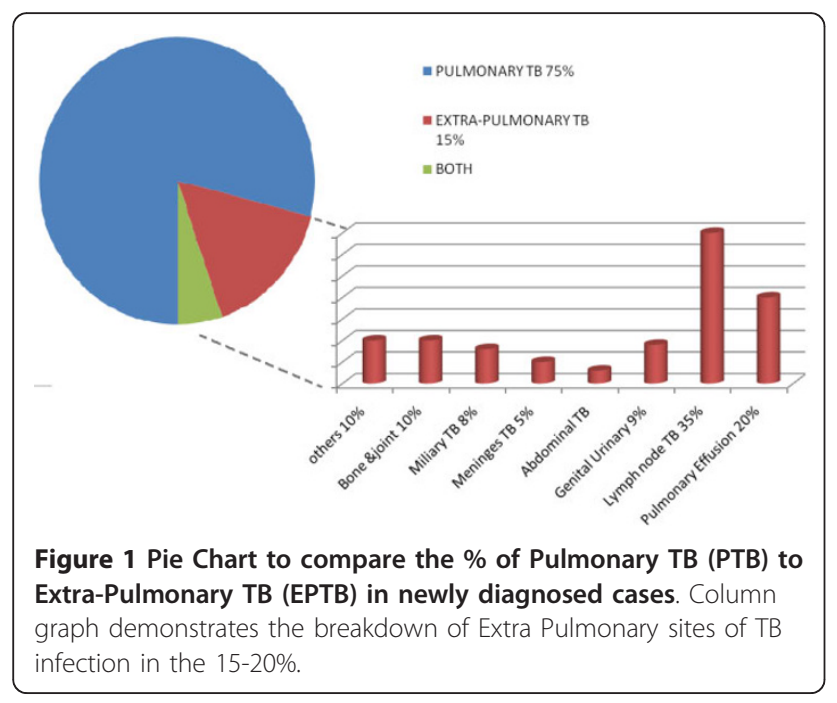

particular site and lie dormant for decades before causing disease.

\section{Case presentation}

An 89-year-old Caucasian woman presented to our outpatients clinic with a left-sided neck lump which she had had for six months. It had started as a small peasized lump and developed into a $3 \mathrm{~cm} \times 4 \mathrm{~cm}$ smooth, fixed mass (Figure 2). It had grown relatively slowly and the woman has been otherwise healthy throughout. She reported no pyrexia, dysphagia, weight loss, night sweats or hoarseness. Accompanied by her niece, our patient made it clear that, if it was malignant, she wanted it treated conservatively.

A further history revealed that she had a three-year history of recurrent neck swellings that progressed to abscesses, continuously discharging and healing slowly.

On closer physical inspection, there was visible scarring from a previous abscess approximately $5 \mathrm{~cm}$ inferior to this mass on her left side. As can be seen in Figure 3 and Figure 4, on our patient's right side there was an old, slowly healing abscess that has been present for over a year.

Our patient had always enjoyed good health, with the only other significant past medical history being a

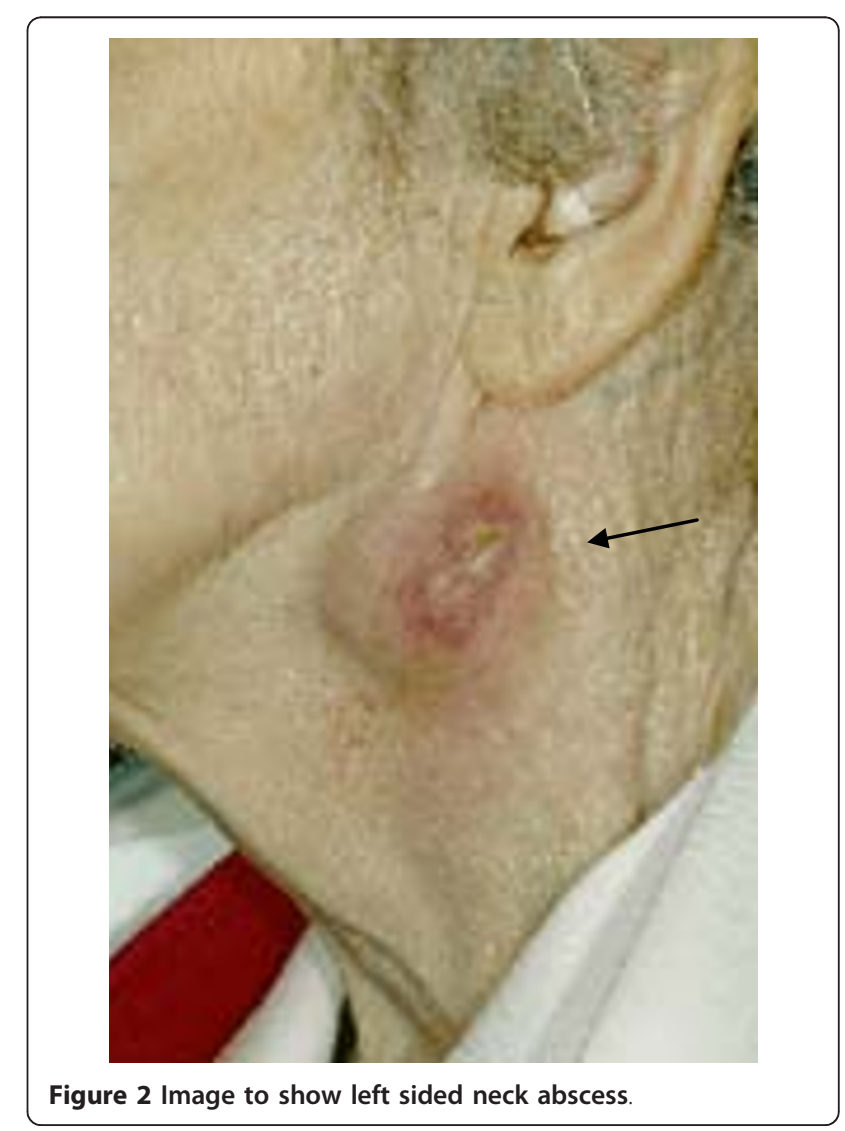




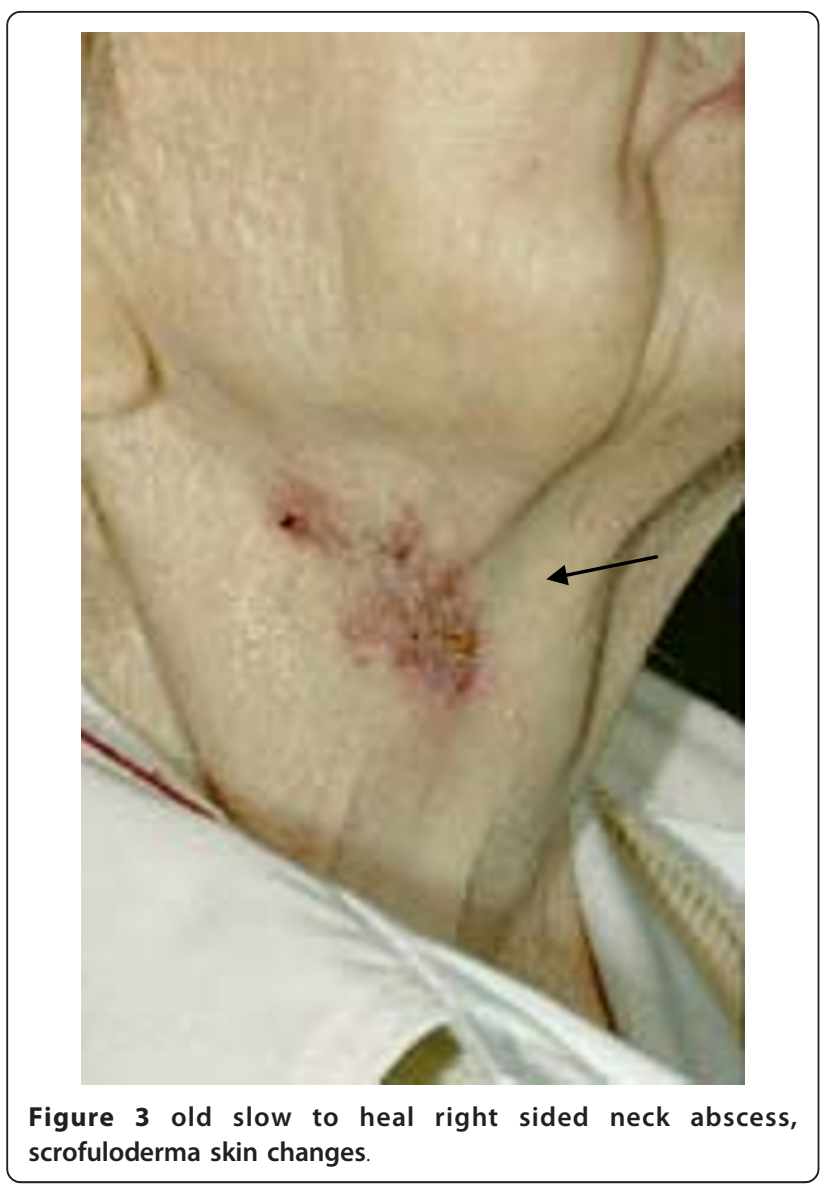

splenectomy, resulting in taking life-long daily penicillin. The abscesses were treated by her general practitioner with recurrent antibiotics and frequent dressings. Previous swabs from the neck abscess, sent by her general practitioner, did not grow the tuberculous organism.

Our patient agreed to have further investigations. A chest X-ray and fine needle aspiration (FNA) were performed. The chest X-ray showed chronic fibrotic changes and no evidence of TB infection. The FNA contained necrotic debris and foam cells; acute inflammatory cells were not prominent and granulomata were not seen. Ziehl-Neelsen staining was performed and no acid-alcohol fast bacilli were seen. The features found represented a partially treated skin abscess but the negative staining did not exclude the possibility of TB. Part of the aspirate was submitted to microbiology for conventional and TB culture.

The differential diagnosis included neoplasia, tertiary syphilis, deep fungi (for example, sporotrichosis, actinomycosis) and chronic granulomatous disease.

Three weeks after submitting the aspirate to microbiology for culture, TB was confirmed. The infectious disease team took over our patient's care immediately. Our patient underwent various tests, including liver

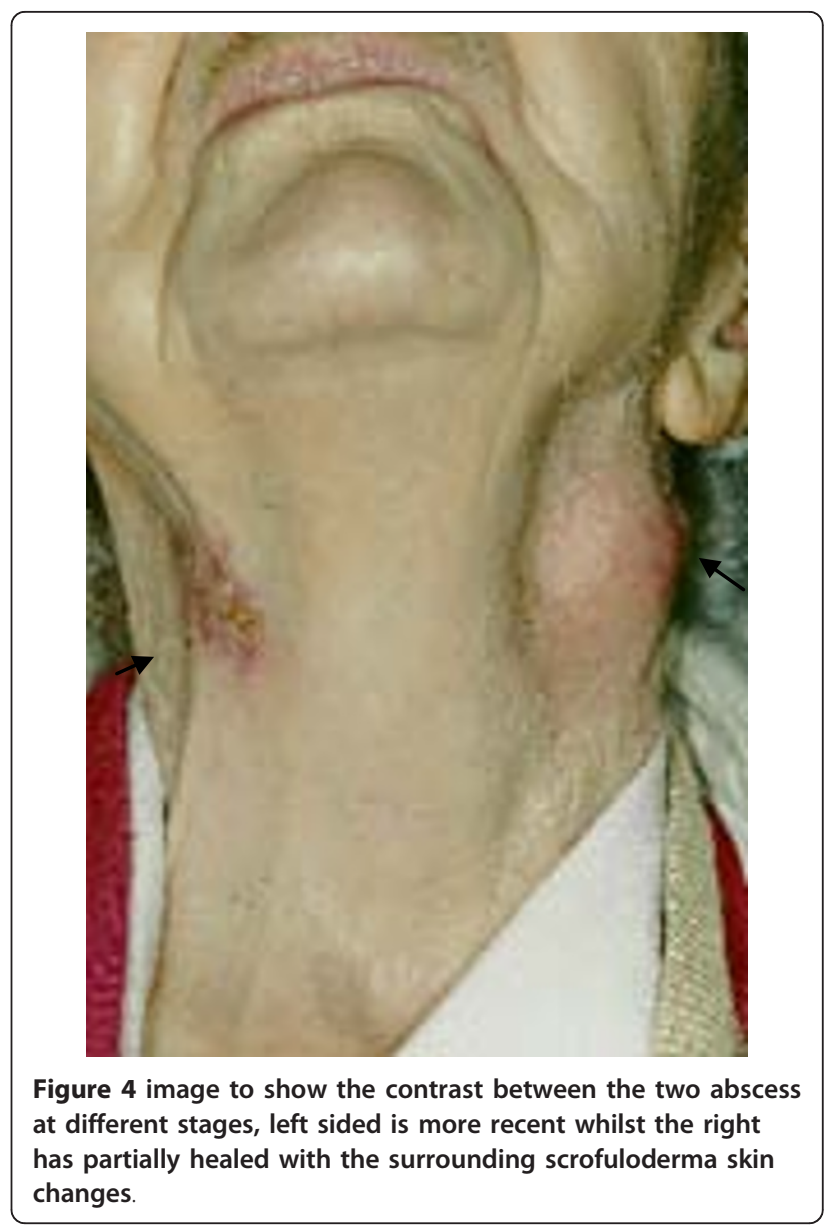

function tests, urea and electrolytes test, HIV screening and visual acuity prior to commencing the anti-TB regime.

Our patient was started on ethambutol $700 \mathrm{mg}$ to be taken in the morning, rifampicin $450 \mathrm{mg}$, isoniazid 300 $\mathrm{mg}$, pyrazinamide $900 \mathrm{mg}$ and pyridoxine $20 \mathrm{mg}$. Within two months she showed a significant improvement, and after four months there were visible scars only.

\section{Discussion}

The body's initial immune response involves Type 1 $\mathrm{CD} 4+\mathrm{T}$ lymphocytes and natural killer $\mathrm{T}$ lymphocytes (NK cells) that secrete interferon-gamma. This activates macrophages to produce a variety of substances that inhibit growth and kill mycobacteria. This is a very simplified explanation; the process involves many other aspects including suppression from interleukin 4-10, amplification by interleukin 12 and many cytokines [5].

Re-infection is a rare event when immunity is intact, however, over the past few decades it has been demonstrated in patients with advanced HIV, with the use of chemotherapy and disease-modifying antirheumatic 
drugs and, in theory, is possible in any other process that reduces the host immune response [6].

Aging is known to have detrimental effects on the immune system and is referred to as immunosenescence. It is a complex process that affects cell mediated immunity [7]. As we age, we lose lymphoid tissue. T-cell activation is reduced plus a larger percentage of activated $\mathrm{T}$-cells responses start later and stop sooner. NK cell activity is also reduced significantly. It has been suggested that, as we age, the innate response prevails over the adaptive response [8-10].

The immune response to TB greatly relies on T lymphocytes and NK cells and so the aging immune system is much less capable of responding to M. tuberculosis.

The spleen is an important organ in the defense against invading pathogens. It acts as a filtering system, permitting phagocytosis of bacteria by cells in the reticuloendothelial system. The spleen is also an important site capable of producing large quantities of antibodies, which has proven vital in preventing and tackling sepsis. Therefore, the major risk post-splenectomy is that of overwhelming sepsis [11].

The humoral role of the spleen has been well documented and recent studies have shown the spleen to have a significant role in cell mediated immunity. The spleen is an important organ for the differentiation and maturation of stem cells into immunocompetent B-cells. $\mathrm{B}$-cells were once mostly associated with the humoral immune response. However, recent studies have shown a variety of interactions of B-cells with the cellular immune response, which is necessary against infection by M. tuberculosis [12].

In the future, if a mass is suspected for $\mathrm{TB}$, tissue should be attained to help with the diagnosis. As in this case, the initial Ziehl-Neelsen stain came back negative, however the culture was positive. This is a common problem and emphasizes the importance of a high clinical suspicion. Having to repeat FNA is also common, especially if the mass is long standing. Some empirical antibiotics, such as macrolides, have been shown to partly treat mycobacterium organisms, therefore reducing the likelihood of getting positive cultures.

Treatment is similar to that for pulmonary disease, which is with isoniazid, rifampicin, pyrazinamide and ethambutol for two months followed by a longer course of rifampicin and isoniazid. The length of time for treatment has long been debated, with no firm consensus. The duration depends on the patient, the response to treatment, the risk of relapse and the site and tissue involved. Where there is limited lymph node involvement, treatment is usually continued for at least four months. Surgery is usually not implicated. With adequate treatment, clinical remission is practically $100 \%$. It is recommended that people in close contact, such as family members, should undergo testing for TB.

There are numerous investigations to be taken prior to initiating anti-TB treatment. These include a full blood count, a urea and electrolytes test, uric acid analysis, liver function tests, HIV screening, chest X-ray and three sputum acid-fast bacillus smears (to ensure no pulmonary involvement). Visual acuity and fields should be documented prior to treatment.

For the first two months, a regime of four drugs is used: isoniazid $5 \mathrm{mg} / \mathrm{kg}$, rifampicin $10 \mathrm{mg} / \mathrm{kg}$, pyrazinamide $20 \mathrm{mg} / \mathrm{kg}$, ethambutol $25 \mathrm{mg} / \mathrm{kg}$ and pyridoxine $10 \mathrm{mg}$ once daily.

Thereafter, a three-weekly regime can be used for a following four or more months: isoniazid $10 \mathrm{mg} / \mathrm{kg}$, rifampicin $10 \mathrm{mg} / \mathrm{kg}$ and pyridoxine $10 \mathrm{mg}$ once daily [13]. Local infectious disease departments should be contacted for advice and risk stratification.

\section{Conclusion}

Our patient and her family were convinced there was an underlying malignant process and therefore she lived with these recurrent lumps and abscesses for years, undergoing daily dressings and multiple courses of antibiotics. She was very relieved to get a diagnosis of neck lymph node TB and overwhelmed to learn that the treatment basically has a $100 \%$ success rate. There is a high false negative culture result from superficial swabs, therefore a biopsy with culture should be considered if there is a high degree of clinical suspicion.

It is important to realize that patients aged over 65 years have waning immunity and therefore are a vulnerable group for infections as well as re-activation. Postsplenectomy patients are at a major disadvantage in sepsis and also in cellular immunity, as in the required response for M. tuberculosis infection.

\section{Consent}

Written informed consent was obtained from the patient as well as her next of kin for the publication of this manuscript and any accompanying images. A copy of the written consent is available for review by the Editorin-Chief of this journal.

\section{Competing interests}

The author declares that they have no competing interests.

Received: 6 December 2010 Accepted: 20 December 2011 Published: 20 December 2011

\section{References}

1. Antony SJ, Harrell V, Christie JD, Adams HG, Rumley RL: Clinical differences between pulmonary and extrapulmonary tuberculosis: a 5-year retrospective study. J Natl Med Assoc 1995, 87(3):187-192.

2. Braun-Falco O, Plewig G, Wolff HH: Dermatology and venereology [in German]. 3 edition. Berlin, Germany: Springer-Verlag; 1984. 
3. Sharma SK, Mahon A: Extrapulmonary Tuberculosis. Indian J Med RES 120 2004, 316-353.

4. Dannenberg AM Jr: Roles of cytotoxic delayed-type hypersensitivity and macrophage-activating cell-mediated immunity in the pathogenesis of tuberculosis. Immunobiology 1994, 191:461-473.

5. Schluger NW, Rom WN: The host immune response to tuberculosis. Am J Respir Crit Care Med 1998, 157(3):679-691.

6. Bhatt K, Salgame P: Host innate immune response to Mycobacterium tuberculosis. Clin Immunol 2007, 29(4):347-362.

7. Boren E, Gershwin ME: Inflamm-aging: autoimmunity, and the immunerisk phenotype. Autoimmun Rev 2004, 3(5):401-406.

8. Yan J, Greer JM, Hull R, O'Sullivan JD, Henderson RD, Read SJ, McCombe PA: The effect of ageing on human lymphocyte subsets: comparison of males and females. Immun Ageing 2010, 7:4.

9. Jiang J, Gross D, Elbaum P, Murasko DM: Aging affects initiation and continuation of T cell proliferation. Mech Ageing Dev 2007, 128:332-339.

10. Schaaf HS, Collins A, Bekker A, Davies PD: Tuberculosis at extremes of age. Respirology 2010, 15(5):747-763.

11. Rozing J, Brons NHC, Benner R: Effects of splenectomy on the humoral immune system, A study in neonatally and adult splenectomized mice. Immunology 1978, 34(5):909-917.

12. Maglione PJ, Chan J: How B cells shape the immune response against Mycobacterium tuberculosis. Eur J Immunol 2009, 39(3):676-686.

13. Yew W, Leung C: Update on the management of pulmonary and extrapulmonary tuberculosis. The Hong Kong Medical Diary. NO.1 2006.

doi:10.1186/1752-1947-5-584

Cite this article as: Niblock: Recurrent neck abscesses due to cervical

tuberculous lymphadenopathy in an elderly woman post-splenectomy: a case report. Journal of Medical Case Reports 2011 5:584.

\section{Submit your next manuscript to BioMed Central} and take full advantage of:

- Convenient online submission

- Thorough peer review

- No space constraints or color figure charges

- Immediate publication on acceptance

- Inclusion in PubMed, CAS, Scopus and Google Scholar

- Research which is freely available for redistribution

Submit your manuscript at www.biomedcentral.com/submit
Ciomed Central 